\title{
Persimmon fruit causing simultaneous small bowel and stomach obstruction
}

Singapore Med J 2019; 60(10): 550 https://doi.org/10.11622/smedj.2019132

Dear Sir,

Persimmons contain large amounts of soluble tannin, which may interact with gastric acid to form a conglomerate that is harder than other phytobezoars. ${ }^{(1)}$ The presence of ingestible fruit tannins, cellulose, hemicellulose and lignins in persimmons make such a phytobezoar more difficult to dissolve or segment into smaller divisions due to its hard consistency. ${ }^{(2)}$ This makes persimmon phytobezoars particularly difficult to manage. We report a case of multiple persimmon phytobezoars causing simultaneous small bowel and gastric outlet obstruction.

A 39-year-old woman was admitted to our hospital with symptoms of nausea, vomiting and epigastric pain. She had no past medical illness. On examination, she had a distended abdomen without scars and no external hernias. Blood investigations and imaging established a diagnosis of intestinal obstruction. Computed tomography established a diagnosis of small bowel intestinal obstruction with grossly distended stomach. There was no reporting of 'bezoar' by the on-duty radiologist. However, the surgical team adjudged the possibility of an intraluminal mass in the distal bowel and retrospectively asked the patient about her dietary history. She confirmed that she had eaten four persimmons from her own farm ten days ago. A clinical diagnosis of small bowel obstruction secondary to persimmon phytobezoar was established.

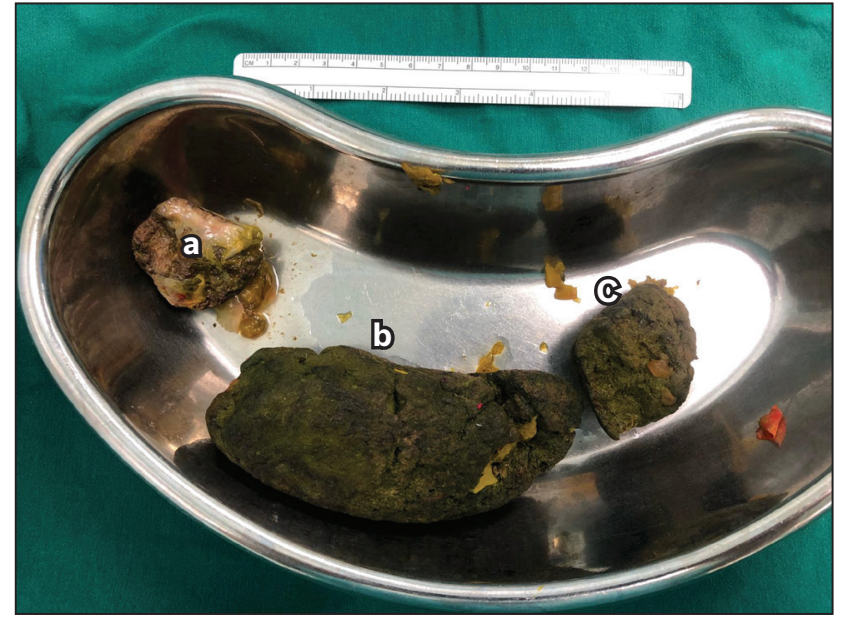

Fig. 1 Photograph shows gross specimens of a $5-\mathrm{cm}$ phytobezoa obstruction in the distal small bowel retrieved through an enterotomy and primary repair $(\mathrm{a} \& \mathrm{c})$. Another $15 \mathrm{~cm} \times 6 \mathrm{~cm}$ sausage-shaped phytobezoar (b) was also imaged and retrieved from the stomach via gastrotomy and primary repair.

\begin{abstract}
chactive management option, as it avoids surgical intervention and
attractive management option, as it avoids surgical intervention and
\end{abstract}

A 5-cm phytobezoar obstruction in the distal small bowel was retrieved through an enterotomy with primary repair. Another $15 \mathrm{~cm} \times 6 \mathrm{~cm}$ sausage-shaped phytobezoar was retrieved from the stomach through a gastrotomy and primary repair (Fig. 1). We postulated that the small bowel phytobezoar in our patient represented the 'daughter' phytobezoar from the 'parent' giant gastric phytobezoar. Her postoperative recovery was uncomplicated, and the patient remained well at the sixmonth follow-up.

Old age, poor dentition, previous gastrectomy, diabetes mellitus and hypothyroidism can increase the risk of bezoar formation due to problems with digestion or gut motility. The clinical manifestation of the bezoar is dependent on the location of the obstruction. Small bowel obstruction is the most common manifestation. The majority of phytobezoars pass spontaneously $(80 \%-90 \%)$; some can be retrieved through an endoscopy $(10 \%-20 \%)$ and surgery is rarely needed $(<1 \%))^{(3,4)}$ Recently, the use of effervescent aerated drinks such as Coca-Cola has been reported in the management of phytobezoars. ${ }^{(5)}$ This is an the occurrence of secondary 'daughter' phytobezoars.

In our patient, endoscopy and Coca-Cola were not advocated due to her small bowel obstruction. In patients with multiple bezoars causing intestinal obstruction, laparotomy is considered more effective to concurrently remove all the bezoars. One should also check for the possibility of more bezoars to avoid early recurrent obstruction from the 'missed' bezoars. Laparoscopic surgery is an option when the expertise is available, but we caution against this approach, as potential concurrent bezoars can be missed due to loss of tactile feedback.

Yours sincerely,

Thomas Zheng Jie Teng ${ }^{1}$, Yen Pin $\underline{T a n}^{2}$, Vishal G $\underline{\text { Shelat }}^{3}$

${ }^{1}$ Lee Kong Chian School of Medicine, Nanyang Technological University, ${ }^{2}$ Department of General Surgery, ${ }^{3}$ Hepatobiliary and Pancreatic Surgery Unit, Department of General Surgery, Tan Tock Seng Hospital, Singapore. ZTENG004@e.ntu.edu.sg

\section{References}

1. Tan F, Mo H, He X, Pei H. An unusual case of gastric outlet obstruction caused by multiple giant persimmon phytobezoars. Gastroenterol Rep (Oxf) 2016 ; pii: gow042.

2. Ben-Porat T, Sherf Dagan S, Goldenshluger A, Yuval JB, Elazary R. Gastrointestinal phytobezoar following bariatric surgery: systematic review. Surg Obes Relat Dis 2016; 12:1747-54.

3. Shelat VG, Tan J. Durian seed causing small bowel obstruction. Dig Surg 2009; 26:212.

4. Ong MW, Wei-Wen A, Shelat VG. Endoscopic management of octopus food bolus lodged within the esophagus. Clin Gastroenterol Hepatol 2018; pii: S1542-3565(18)30368-9.

5. Eng K, Kay M. Gastrointestinal bezoars: history and current treatment paradigms. Gastroenterol Hepatol (N Y) 2012; 8:776-8. 